\title{
Electrostatic Characteristics of High-k Stacked Gate-All-Around Heterojunction Tunnel Field Effect Transistor using Superposition Principle
}

usha C ( $\square$ usha.chintu.dec14@gmail.com )

Dayananda Sagar College of Engineering https://orcid.org/0000-0003-2035-7878

\section{P Vimala}

Dayananda Sagar College of Engineering

K Ramkumar

Vellore Institute of Technology: VIT University

\section{V.N. Ramakrishnan}

Vellore Institute of Technology: VIT University

\section{Research Article}

Keywords: Gate All Around Tunnel Field Effect Transistor, Heterojunction, Superposition Principle, Surface Potential, Electric Field, Drain Current and TCAD

Posted Date: August 6th, 2021

DOI: https://doi.org/10.21203/rs.3.rs-708302/v1

License: (c) (i) This work is licensed under a Creative Commons Attribution 4.0 International License. Read Full License 


\title{
Electrostatic Characteristics of High-k Stacked Gate-All-Around Heterojunction Tunnel Field Effect Transistor using Superposition Principle
}

\author{
${ }^{* 1}$ Dr.Usha.C, ${ }^{2}$ Dr.P.Vimala, ${ }^{3}$ K.Ramkumar, ${ }^{4}$ Dr. V.N. Ramakrishnan \\ ${ }^{1,2}$ Department of Electronics and Communication, Dayananda Sagar College of Engineering, \\ Bangalore-560078 \\ ${ }^{3,4}$ School of Electronics Engineering, Vellore Institute of Technology, Vellore-632014 \\ E-mail: ksr.cusha@gmail.com, ervimala@gmail.com, ramkumar.ece2009@gmail.com
} And vnramakrishnan@vit.ac.in

\begin{abstract}
We use superposition method to model the electrostatic characteristics of high-k stacked Gate-All-Around Hetero Junction TFETs (GAA-HJTFETs). The hetero junction is set up by using $\mathrm{Ge} / \mathrm{Si}$ material in the source/channel respectively. The modeling is accomplished by considering the space charge regions at the source-channel/drainchannel junctions and the channel region. The surface potential in the channel region is obtained by applying superposition principle, where as in source/drain it is derived by solving 2-D/1-D Poisson's equation respectively. Furthermore, the electric field and drain current are modeled from the surface potential and Kane model respectively. The results are confirmed using ATLAS TCAD simulation.
\end{abstract}

\section{Keywords}

Gate All Around Tunnel Field Effect Transistor, Heterojunction, Superposition Principle, Surface Potential, Electric Field, Drain Current and TCAD

\section{Introduction}

With years of continuous down-scaling the transistors in CMOS technology leads to deteriorate the subthreshold swing (SS), leakage current and threshold voltage (VT) [1-3]. In addition, the power crisis faced by the device engineers, entail the gateway to the multi-gate/gate-all-around FETs, group III-V based FETs, super steep subthreshold slope FETs, and graphene/carbon-nanotube based FETs . Tunnel FETs is one such device which earned the awareness of researchers, because of its low sub-threshold swing $(<60$ $\mathrm{mV} /$ decade) and reduced short channel effects $[4,5]$. A three-terminal $p-i-n$ device, where the source and drain are doped contrary, besides the channel is either intrinsic or lightly doped is called TFETs. It has two junctions namely, drain-channel junction (JDC) and source-channel (JSC). The carrier transport in TFETs is controlled by inter-band tunneling (BTBT), while thermionic emission in Metal Oxide Semiconductor FETs (MOSFETs). Though the device exhibits low SS and Ioff, but also suffers from low ION and ambipolar conduction $[6,7]$. In this regard, alternative approach such as gate engineering (i.e., employing of multi-gate/gate-all-around structures) [8-10], gate dielectric engineering (i.e., make use of high-k material as a gate dielectric) [11], work function engineering (i.e., employing asymmetric gate work 
functions instead of symmetric) [12], tunnel engineering (i.e., bring in a heavily doped source pocket at the JSC such that its completely depleted) [13] and materials engineering (i.e., using lower band-gap materials like InAs, GaAs, InGaAs, SiGe and $\mathrm{Ge}$ as an alternative to $\mathrm{Si}$ ) [14] mechanisms have been proposed to mitigate the shortcomings of TFETs.

Over the decades, quite a few analytical models for the electrical parameters in particular surface potential, electric field, threshold voltage and drain current for single/double gate and GAATFETs [15-17] with single/dual material gate (SMG/DMG) electrode were reported. The electrical outcomes of the aforementioned devices were examined by Verhulst et al. [15]. The 2-D electrostatic response of DG and GAA TFETs were reported by Pan et al. [16]. Vishnoi et al. [17] proposed the BTBT current model for DG-GAA-TFETs irrespective of the depletion regions at JSC and JDC by pseudo-2-D analytic modeling. The DMG in DG-TFETs exhibits better drive current and SS than SMG counterpart as reported by Kumar et al. [18] and Jain et al. [19]. Kumar et al. [20] and Prabhat et al. [21] reported the modeling of drive current and surface potential for DMG with SG/DG TFET structures respectively. The device performance by can be enhanced by replacing $\mathrm{SiO} 2$ with pile up $\mathrm{SiO} 2$ and a high$\mathrm{k}$ dielectric in DG TFETs as reported by Kumar et al [22]. The proposed Ge-Si-Si hetero stacked gate dielectric GAA-TFET device provides better ION per unit area than its counterpart planner devices. Hence, developing an accurate model for surface potential, electric field and drain current becomes important.

The corroboration of this model is verified against previously published reports and 3Dnumerical simulators [23]. The paper is presented as follows: Section 2 and 3 discuss about the device architecture and results followed by conclusion.

\section{Device Structure}

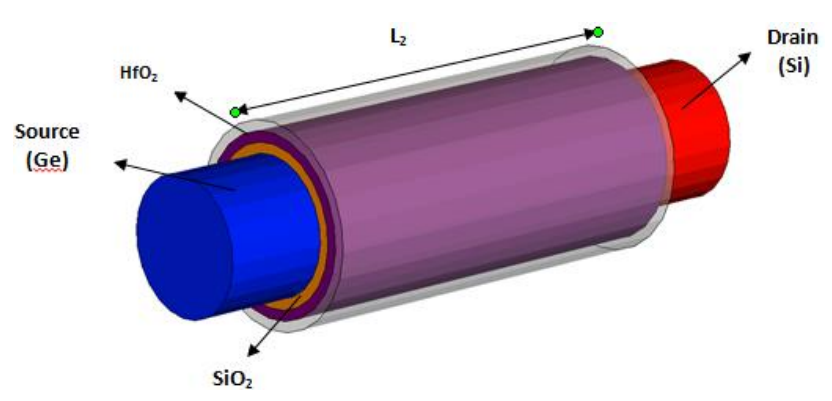

Fig. 1a. 3-D view of high-k stacked GAA HJ-TFET

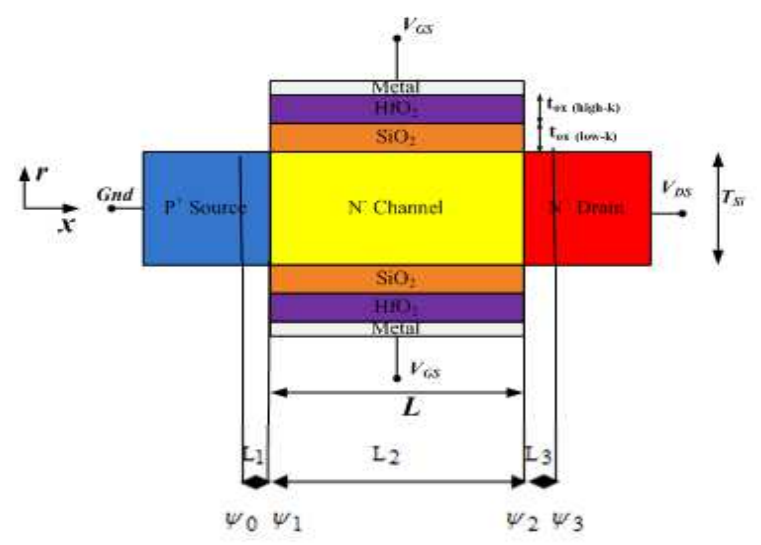

Fig. 1b. Cross sectional 2-D view of high-k stacked GAA HJ-TFET

Fig. 1(a) and Fig. 1(b) shows the 3-D and 2-D view of proposed TFETs, where the high-k dielectric is deposited on top of the low-k dielectric. The device specifications are tabulated in the table 1 . Three depletion regions are considered for modeling namely region I, II and III are source-channel, channel and drain-channel respectively. The length of three corresponding regions are considered as $L_{1}, L_{2}$ and $L_{3}$. The potentials across the corresponding regions are $\psi_{0}, \psi_{1}$, $\psi_{2}$ and $\psi_{3}$. 
Table 1. List of parameters for High-k stacked GAA HJ-TFET

\begin{tabular}{|c|c|c|c|}
\hline Sl.N & Symbo & Description & Value \\
\hline 1 & $\mathrm{~N}_{\mathrm{S}}$ & Source & $1 \times 10^{20}$ \\
\hline 2 & $\mathrm{~N}_{\mathrm{C}}$ & Channel & $1 \times 10^{16}$ \\
\hline 3 & $\mathrm{~N}_{\mathrm{D}}$ & Drain & $5 \times 10^{18} \mathrm{c}$ \\
\hline 4 & $\mathrm{~L}_{2}$ & Length of the & $50 \mathrm{~nm}$ \\
\hline 5 & $\phi_{\mathrm{m}}$ & Work function & $4.3 \mathrm{eV}$ \\
\hline 6 & $\mathrm{t}_{\mathrm{ox}}$ & Thickness of & $1 \mathrm{~nm}$ \\
\hline 7 & $\mathrm{t}_{\mathrm{k}}$ & Thickness of & $2 \mathrm{~nm}$ \\
\hline 8 & $\mathrm{t}_{\mathrm{si}}$ & Thickness of Si & $13 \mathrm{~nm}$ \\
\hline 9 & $\varepsilon_{\mathrm{o}}$ & Permittivity of & $8.854 \times 10$ \\
\hline 10 & $\varepsilon_{\mathrm{si}}$ & Permittivity of & $11.9 \varepsilon_{\mathrm{o}}$ \\
\hline 11 & $\varepsilon_{\mathrm{OX}}$ & Permittivity of & $3.9 \varepsilon_{\mathrm{o}}$ \\
\hline
\end{tabular}

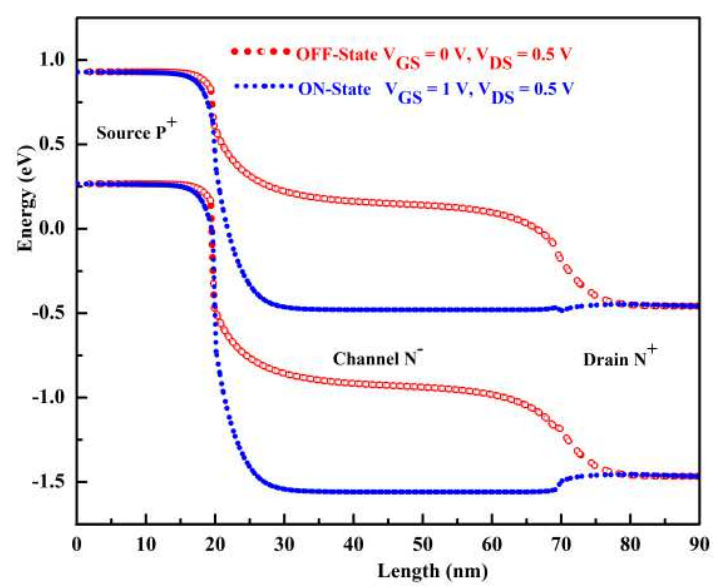

Fig. 2. Energy Band profile of proposed

GAA HJ-TFET

Fig. 2. depicts the band profile of the proposed device. When $\mathrm{V}_{\mathrm{GS}}=0 \mathrm{~V}$ (OFFState) the inter-band tunneling is inhibited, as a result no electrons tunnel through the depletion region at $\mathrm{J}_{\mathrm{SC}}$. When $\mathrm{V}_{\mathrm{GS}}=1 \mathrm{~V}$ (ON-State) the energy band gap reduces and inter-band tunneling is permitted which allows the electrons to tunnel from the source to channel. The tunneling rate of electrons is high in hetero-junction devices compared to homo-junction devices due to narrow bandgap materials at source.

\section{MODEL DERIVATION}

Figure 1(b) shows the 2-D view of the device considered for modeling. The co-ordinates of the device were denoted by $\mathrm{z}$ and $\mathrm{r}$ - axes and the junction potentials were given by $\psi_{0}, \psi_{1}$, $\psi_{2}$ and $\psi_{3}$ at $z=0, z_{1}=L_{1}, z_{2}=L_{1}+L_{2}$ and $z_{3}=L_{1}+L_{2}+L_{3}$.

\subsection{Modeling of Potential in the channel}

The distribution of potential in the gate all around device is same as the double gate device structure. The tubular symmetry nature of the proposed device leads to an angular co-ordinate independent of surface potential. Thus, the 2-D Poisson's equation of cylindrical co-ordinates is considered for modeling and in channel region it is given by

$$
\frac{1}{r} \frac{\partial}{\partial r}\left(r \frac{\partial \psi_{c h(z, r)}}{\partial r}\right)+\frac{\partial^{2} \psi_{c h}(z, r)}{\partial r^{2}}=\frac{q}{\varepsilon_{s i}} \eta(1)
$$

Where $\psi_{c h}(z, r)$ is potential across the channel, $\varepsilon_{s i}$ - permittivity of silicon. The charge density of mobile carriers across the channel is given as

$\eta=\eta_{i} \exp \left(\frac{\psi \operatorname{ch}(z, r)-V(z, r)}{V_{T}}\right)$

Where $V_{T}=\frac{K T}{q}$ the thermal voltage at 300 $\mathrm{K}$ and $\mathrm{V}$ the non-equilibrium quasi-Fermi level referred to Fermi level of source region with the boundaries given by [24].

$V(\mathrm{O}, r)=\mathrm{O}$

$V\left(L_{2}, r\right)=V_{D S}$

The quasi-Fermi level is almost constant in the radial direction [25]. $\mathrm{V}_{\mathrm{DS}}$ is approximated to be drain-source voltage along the channel expect at the left verge of the channel. At the channel electrostatic potential boundary conditions are 
$C_{d}\left[V_{G S}-\phi_{m s 1, i}-\psi_{c h}\left(z, r=r_{0}\right)\right]=\left.\left.\varepsilon_{s i} \frac{\partial \psi_{c h}(z, r)}{\partial r}\right|_{r=r_{0}} \frac{d \varphi_{1}(r)}{d r}\right|_{r=0}=0$

$\psi_{c h}(0, r)=\phi_{0 s}$

$\psi_{c h}(L, r)=\phi_{0 d}$

Where $C_{d}$ the capacitance dielectric per unit Using equation (12) and (13), 1-D potential is obtained as

$\varphi_{1}(r)=V+U_{T} \ln \left(\frac{8 B \varepsilon_{s i}}{q n_{i}\left(B r^{2}+1\right)^{2}}\right)$ area.

$$
C_{d}=\frac{\varepsilon_{O x}}{r_{0} \ln \left(1+\frac{t_{O x}}{r_{0}}\right)}
$$

Where $r_{0}=\frac{t_{s i}}{2}, \phi_{m s 1, i}$ the work function of Substituting equation (14) in equation (12), we can determine $\mathrm{B}$

$$
\frac{V_{G S}-\phi_{m s}-V}{U_{T}}-\ln \left(\frac{8 B \varepsilon_{s i}}{q n_{i}\left(B r_{0}^{2}+1\right)^{2}}\right)=-\frac{4 B U_{T} r}{\operatorname{Cox}\left(B r^{2}+1\right)}
$$

gate referenced to the silicon material

$\phi_{m s 1, i}=\phi_{m}-\left(\chi_{s i}+\frac{E_{g s i}}{2 q}\right)$

$\phi_{m}, \chi_{s i}, E_{g s i}, \phi_{o s}$ and $\phi_{o d}$ are the work function of gate, electron affinity of $\mathrm{Si}$, energy band gap of $\mathrm{Si}$, the left end and right end potentials respectively.

$\psi_{c h}(z, r)=\varphi_{1}(r)+\varphi_{2}(z, r)$

Where $\varphi_{1}(r)$ is the solution of 1-D Poisson's equation obtained as shown below

$\frac{1}{r} \frac{\partial}{\partial r}\left(r \frac{\partial \varphi_{1}(r)}{\partial r}\right)=\frac{q}{\varepsilon_{S i}} n_{i} \exp \left(\frac{\varphi_{1}(r)-V}{V_{T}}\right)$

The boundary condition at the interface of silicon-dielectric is

$$
C_{o x}\left[V_{G S}-\phi_{m s}-\varphi_{1}\left(r=r_{0}\right)\right]=\left.\varepsilon_{s i} \frac{d \varphi_{1}(r)}{d r}\right|_{r=r_{0}}
$$

Considering the symmetric of the structure by

From equation (10) the solution $\varphi_{2}(z, r) 2-\mathrm{D}$ potential equation is obtained from residual 2-D equation

$$
\nabla^{2} \varphi_{2}(z, r)=\frac{q}{\varepsilon_{s i}} \times n_{i} \exp \left(\frac{\varphi_{1}(r)-V}{U_{T}}\right) \times\left[\exp \left(\frac{\varphi_{2}(z, r)}{U_{T}}\right)-1\right]
$$

The boundary conditions to be satisfied by $\varphi_{2}(z, r)$ are [24]

$$
\begin{aligned}
& \varphi_{2}(0, r)=\varphi_{O S}-\varphi_{1}(r) \\
& \varphi_{2}(L, r)=\varphi_{O D}-\varphi_{1}(r) \\
& C_{O x}\left[-\varphi_{2}\left(z, r=r_{0}\right)\right]=\left.\varepsilon_{S i} \frac{\partial \varphi_{2}(z, r)}{\partial r}\right|_{r=r_{0}}
\end{aligned}
$$

Assuming $\varphi_{2} / U_{T}$ is small equation (16) is reduced to 2-D Laplace equation. This approximation is valid in TFETs [26]. The separation of variable method is used to derive the $\varphi_{2}(z, r)$. The expression attained is

$$
\varphi_{2}(z, r)=\left[C_{0} e^{\frac{\lambda}{r_{0}} z}+C_{1} e^{-\frac{\lambda}{r_{0}} z}\right] J_{0}(\lambda, r)
$$


where $J i(x)$ is the first kind Bessel function of the order $i$. Using (20) in (18) gives the separation factor $\lambda$ relation (should be a positive value)

$$
C_{o x} \frac{r_{0}}{\varepsilon_{s i}}=\lambda \frac{J_{1}(\lambda)}{J_{0}(\lambda)}
$$

Consider $C_{r}=C_{o x} \frac{r_{0}}{\varepsilon_{s i}}$. Applying the boundary conditions (17) and (18) in equation (20) to obtain the expression for the co-efficient of equation (20) (detailed derivation is given in Appendix).

$$
\begin{gathered}
C_{0}=\frac{1}{2 N \operatorname{Sinh}\left(\frac{\lambda L}{r_{0}}\right)}\left[\frac{J_{1}(\lambda)}{\lambda}\left(\phi_{o D}-\phi_{0 S}\right) \exp \left(-\frac{L \lambda}{r_{0}}\right)\right. \\
\left.+S_{1} V_{a}\right] \\
C_{1}=\frac{1}{2 N \operatorname{Sinh}\left(\frac{\lambda L}{r_{0}}\right)}\left[\frac{J_{1}(\lambda)}{\lambda}\left[\varphi_{0 S} \exp \left(\frac{L \lambda}{r_{0}}\right)-\varphi_{0 D}\right]\right. \\
\left.+S_{2} V_{a}\right]
\end{gathered}
$$

Based on the device dimensions, $S_{1}, S_{2}$ and $N$ are given by

$$
\begin{aligned}
& S_{1}=\exp \left(-\frac{L \lambda}{r_{0}}\right)-1 \\
& S_{2}=1-\exp \left(\frac{L \lambda}{r_{0}}\right) \\
& N=\frac{J_{0}^{2}(\lambda)}{2}\left[\left(\frac{C_{r}}{\lambda}\right)^{2}+1\right]
\end{aligned}
$$

The total potential across the channel of proposed device is gained by adding the potential terms obtained from equations (14) and (20).
3.2. Modeling of Potential in the depletion region of Source

The depletion region across the source cannot be neglected for higher voltages, thus the voltage drop along this region is to be considered. The 2-D Poisson's equation in region $\mathrm{R} 1$ is given by

$\frac{1}{r} \frac{\partial}{\partial r}\left(r \frac{\partial \varphi_{S}(z, r)}{\partial r}\right)+\frac{\partial^{2} \varphi_{S}(z, r)}{\partial z^{2}}=\frac{q N_{A}}{\varepsilon_{s i}} \quad-L_{1} \leq x \leq 0$

Where $\varphi_{S}(z, r)$ is electrostatic potential that refers to the fermi level in the source region and $N_{A}$ is the doping concentration in the source. The electrostatic potential along the $r$ direction is approximated to parabolic approximation equation [27].

$\varphi_{S}(z, r)=A_{0}(z)+A_{1}(z) r+A_{2}(z) r^{2}$

The boundary conditions to obtain the coefficient of the parabolic approximation equation are

$\varphi_{S}(z, r)=\varphi_{S}(z)$
$\left.\frac{d \varphi_{S}(z, r)}{d r}\right|_{r=0}=0$

$C_{f}\left[V_{G S}-\phi_{m s, i}-\phi_{S}(z)\right]=\left.\varepsilon_{s i} \frac{\partial \varphi_{S}(z, r)}{\partial r}\right|_{r=r_{0}}$

Where $C_{f}=\frac{2}{\pi} C_{o x}$ is the fringing field effect considered by the conformal mapping techniques as[28]. Applying the boundary conditions to equation (28), we obtain the coefficient as

$A_{0}(z)=-\frac{r_{0} C_{O x}}{\pi \varepsilon_{s i}}\left(V_{G S}-\phi_{m s, i}\right)+\left(1+\frac{r_{0} C_{O x}}{\pi \varepsilon_{S i}}\right) \varphi_{S}(z)$

$A_{1}(z)=0$ 


$$
A_{2}(z)=\frac{C_{O x}}{\pi r_{0} \varepsilon_{s i}}\left(V_{G S}-\phi_{m s, i}-\phi_{S}(z)\right)
$$

Substituting $A_{0}(x), A_{1}(x)$ and $A_{2}(x)$ in the parabolic equation (28), we get

$$
\frac{\partial^{2} \phi_{S}(z)}{\partial z^{2}}-\frac{4 C_{o x}}{\pi r_{0} \varepsilon_{s i}} \phi_{S}(x)=\eta
$$

Where $\eta=\frac{q N_{A}}{\varepsilon_{s i}}-\frac{1}{K_{d}^{2}}\left(V_{G S}-\phi_{m s, i}\right)$,

$$
K_{d}=\sqrt{\frac{\pi r_{0} \varepsilon_{s i}}{4 C_{o x}}}
$$

The general solution of equation (35) is

$$
\phi_{S}(z)=B_{0} \exp \left(\frac{z+L_{1}}{K_{d}}\right)+B_{1} \exp \left(-\frac{z+L_{1}}{K_{d}}\right)+\eta
$$

The $\varphi_{S}$ expression has to satisfy the boundary conditions as below

1. $\phi_{S}(0)=\varphi_{0 s}$

2. $\phi_{S}\left(-L_{1}\right)=-V_{T} \ln \left(N_{A} / n_{i}\right)$

3. $\left.\frac{d \phi_{S}(z)}{d z}\right|_{z=-L_{1}}=0$

On applying the above boundary conditions from equation (37) to equation (39) in equation (36), the expression is obtained as

$\phi_{S}(z)=\eta-\left(\left(\eta+V_{T} \ln \left(N_{A} / n_{i}\right)\right) \cosh \left(\frac{z+L_{1}}{K_{d}}\right)\right)(40)$

The length of the depletion region at the source is obtained from the potential model when $\mathrm{z}=0$ in equation (36)

$$
L_{1}=K_{d} \cosh ^{-1}\left(\frac{\eta-\varphi_{0 S}}{\eta-V_{F, S}}\right)
$$

Where $V_{F, S}=-V_{T} \ln \left(\frac{N_{A}}{n_{i}}\right)$, equation (40) represents the depletion length $L_{1}$ depends on the gate to source voltage and through $\eta$ and $\varphi_{0 s}$

\subsection{Modeling of Potential in the depletion region of Drain}

For lower gate voltages the drain length cannot be neglected. Thus the modeling of potential at the drain depletion region is derived by neglecting the radial direction. The 1-D Poisson's equation in region (R3) is given as

$$
\frac{\partial^{2} \varphi_{D}(z)}{\partial z^{2}}=-\frac{q N_{D}}{\varepsilon_{s i}} \quad L_{2} \leq z \leq L_{2}+L_{3}
$$

Where $\varphi_{D}(z)$ is electrostatic potential that refers to the Fermi level in drain region and $N_{D}$ is the doping concentration of drain region. The fringing field is ignored in this modeling. The boundary conditions are

$$
\begin{aligned}
& \text { 1. } \varphi_{D}\left(L_{2}\right)=\varphi_{0 D} \\
& \text { 2. } \varphi_{D}\left(L_{2}+L_{3}\right)=V_{D S}+V_{T} \ln \left(\frac{N_{D}}{n_{i}}\right)
\end{aligned}
$$

On integrating equation (41) twice and applying boundary condition the following expression is obtained as

$$
\begin{aligned}
\varphi_{D}(z)= & -\frac{q N_{D}}{2 \varepsilon_{s i}}\left(z-L_{1}-L_{3}\right)^{2}+ \\
& \left(\frac{V_{F, D}-\varphi_{0 D}}{L_{3}}-\frac{q N_{D} L_{3}}{2 \varepsilon_{s i}}\right)\left(z-L_{2}-L_{3}\right) \\
& +V_{F, D}
\end{aligned}
$$

Where $\quad V_{F, D}=V_{D S}+V_{T} \ln \left(N_{D} / n_{i}\right)$. The electric field in lateral direction approaches to zero at the right edge of drain depletion region. 
$\left.\frac{d \varphi_{D}(z)}{d z}\right|_{z=L_{2}+L_{3}}=0$

The depletion length at the drain region is gained by the potential model

$$
L_{3}= \pm \sqrt{\frac{2 \varepsilon_{S i}}{q N_{D}}\left(V_{F, D}-\varphi_{0 D}\right)}
$$

\subsection{Modeling of Drain Current}

The drain current modeling is derived using Kane's Model. The minimum tunneling path length is taken into consideration for calculating tunneling current. The tunneling path is defined as path between conduction band energy point and valance band energy point at the tunneling junction where interband tunneling mechanism takes place. Its length show a discrepancy from shortest tunneling path $\left(l_{\text {short }}\right)$ to longest tunneling path $\left(l_{\text {long }}\right)$.

In Kane's Model, the band BTBT generation rate $\left(G_{B T B T}\right)$ of carriers per unit volume per unit time is given as [29]

$G_{B T B T}=\frac{A_{k a n e}}{\sqrt{E_{g}}} \cdot\left|E_{z, r}\right|^{\alpha} \cdot \exp \left(-B_{k a n e} \cdot \frac{E_{g}^{\frac{3}{2}}}{\left|E_{z, r}\right|}\right)$

Where $\left|E_{z, r}\right|$ is the magnitude of electric field expressed as $\left|E_{z, r}\right|=\sqrt{E_{z}^{2}+E_{r}^{2}}, \alpha$ is 2 for the direct and 2.5 for indirect band gap tunneling, $A_{k a n e}$ and $B_{k a n e}$ are the Kane's constant with values $A_{\text {kane }}=3.5 \times 10^{21} \mathrm{eV}^{0.5} / \mathrm{cm} . \mathrm{s} . \mathrm{V}^{2} \quad$ and $B_{\text {kane }}=2.25 \times 10^{7} \mathrm{~V} / \mathrm{cm} . \mathrm{eV}^{1.5}[29][30]$.

The drain current is derived by integrating $\mathrm{G}_{\text {Втвт }}$ over the tunneling volume [31]

$$
\begin{aligned}
I_{\text {BTBT }}= & \frac{q A_{\text {kane }}}{\sqrt{E_{g}}} \cdot \int_{V} E_{z} \cdot E_{\text {avg }}^{\alpha-1} . \\
& \exp \left(-B_{\text {kane }} \cdot \frac{E_{g}^{\frac{3}{2}}}{E_{\text {avg }}}\right) d V
\end{aligned}
$$

Since equation (47) represents the exponential function of electric field, radial term is neglected in $G_{B T B T}$. The channel lateral electrical field is obtained from the derivative of equation (20), which is expressed as

$$
\begin{aligned}
E_{z}(z, r)= & \frac{\lambda}{r_{0}} \cdot\left[C_{0} \exp \left(\lambda \frac{z}{r_{0}}\right)-C_{1} \exp \left(-\lambda \frac{z}{r_{0}}\right)\right] \cdot \times \\
& J_{0}(\lambda \cdot r)
\end{aligned}
$$

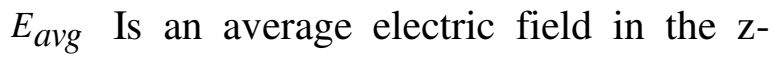
direction over the tunneling path and can be expressed as [31]

$E_{\text {avg }}=\frac{E_{g}}{q L_{T}}$

Where $L_{T}$ is tunneling path length, substituting average and lateral electric field in equation (49)

Yields,

$$
\begin{aligned}
I_{B T B T} & =\frac{2 \pi \lambda q A_{\text {kane }}}{r_{0} \sqrt{E_{g}}} \int_{0 l_{\text {short }}}^{r_{0}} \int_{\text {long }}^{\text {f }}\left[C_{0} \exp \left(\lambda \frac{z}{r_{0}}\right)\right. \\
& \left.-C_{1} \exp \left(-\lambda \frac{z}{r_{0}}\right)\right] J_{0}(\lambda \ldots r) \times \\
& \exp \left(-q B_{\text {kane }} E_{g}^{\frac{1}{2}} . . z\right) \cdot\left(\frac{E_{g}}{q z}\right)^{\alpha-1} d z d r
\end{aligned}
$$

The $I_{B T B T}$ is calculated by integrating equation (52) over the exponential terms due 
rapid change of exponential term than the polynomial term $\left(1 / z^{\alpha-1}\right)$

$$
\begin{aligned}
I_{B T B T}= & \frac{2 \pi \lambda q A_{\text {kane }}}{r_{0} \sqrt{E_{g}}}\left(\frac{E_{g}}{q}\right)^{\alpha-1} \times \\
& {\left[H\left(l_{\text {short }}\right)-H\left(l_{\text {long }}\right)\right] \times\left(\frac{r_{0}}{\lambda}\right) \times . } \\
& J_{1}\left(\lambda . r_{0}\right)
\end{aligned}
$$

Where $H(z)$ is defined as

$$
H(z)=\frac{1}{z^{\alpha-1}}\left(A_{0} \exp \left[\frac{C_{0}}{A_{0}} z\right]+B_{0} \exp \left[-\frac{C_{1}}{B_{0}} z\right]\right)
$$

Where $A_{0}=\frac{C_{0}}{\frac{\lambda}{r_{0}}-q B_{\text {kane }} E_{g}^{\frac{1}{2}}}$ and

$$
B_{0}=\frac{C_{1}}{\frac{\lambda}{r_{0}}+q B_{k a n e} E_{g}^{\frac{1}{2}}}
$$

\subsection{Modeling of transconductance}

The transconductance is derived from drain current as

$$
g_{m}=\frac{\partial I_{B T B T}}{\partial V_{G S}}
$$

\section{Result and Discussion}

In this section, the results are plotted for the analytical modeling and validated using the ATLAS TCAD Simulation tool. The channel length of the proposed model is of $50 \mathrm{~nm}$ with source and drain length of 20 $\mathrm{nm}$. The simulation models used for the simulation of the device are Concentration Dependent, Lombardi Model, Boltzmann, Shockley-Read-Hall, Auger and Band-toBand.

Fig. 3. represents the surface potential profile along the channel length for gate to source voltage of $0 \mathrm{~V}$ and $1 \mathrm{~V}$. It is studied that electron density across the channel increases with increase in gate voltage, especially near the drain region. The extension of drain/source depletion regions is observed at low/high gate voltages. The surface potential profile of model is close to the simulation results. Fig. 4. shows the surface potential profile along the channel length for different silicon thickness $13 \mathrm{~nm}$, $14 \mathrm{~nm}$ and $15 \mathrm{~nm}$. It is observed from plot that higher the thickness of silicon higher the surface potential value. As the thickness of silicon is high the electron density increase, due to which the conductivity increases along the channel, further increases the surface potential value.

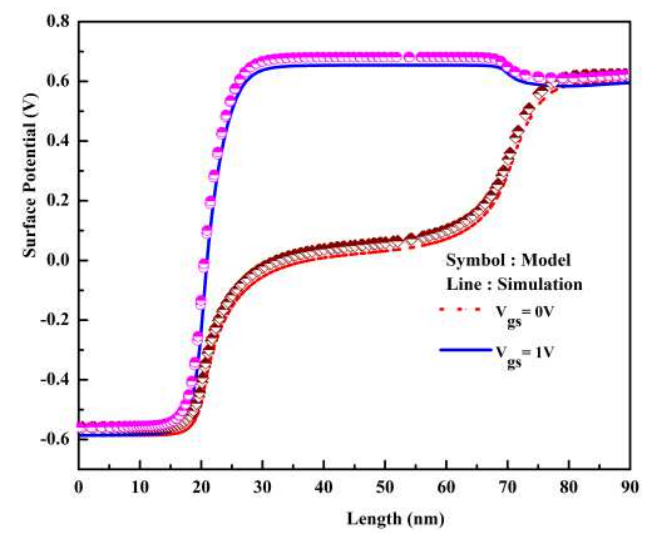

Fig. 3. Surface potential profile along the channel of the TFET for different $V_{G S}$

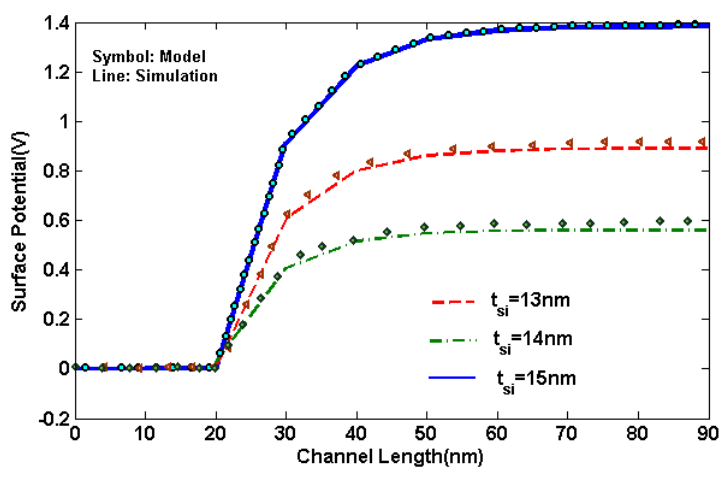

Fig. 4. Surface potential profile for different $\mathrm{t}_{\mathrm{si}}$ 
Fig. 5. depicts the surface potential profile along the channel for different drain voltages of $0 \mathrm{~V}$ and $1 \mathrm{~V}$. Plot represents the variation of potential across the drain region and constant over the source and channel region. As the drain voltage is varied the potential profile changes across the drain region due to increase in carrier density. Fig. 6. comparison plot of lateral electric field profile for with high-k and without high-k GAA HJTFET. It is studied that the high-k stacked GAA HJTFET has higher electric field profile than GAA TFET. The peak across the source and channel region is due to variation of the potential. The peak is low at the drain to channel region of high-k GAA HJ TFET than GAA TFET. Figure 7 shows the comparison of drain current variation with the gate voltage, it is observed that high$\mathrm{k}$ stacked GAA-HJTFET is having higher drain current than GAA-HJTFET due to the higher tunneling rate.

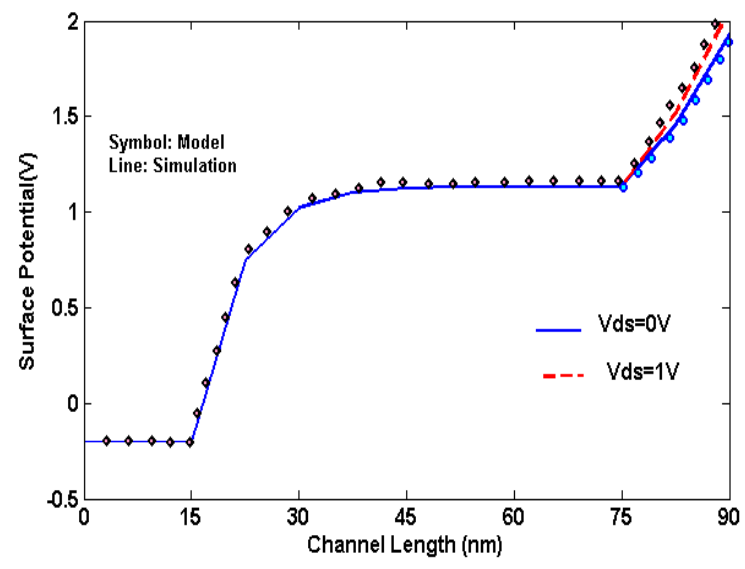

Fig. 5. Surface potential profile along the channel length for different $V_{D S}$

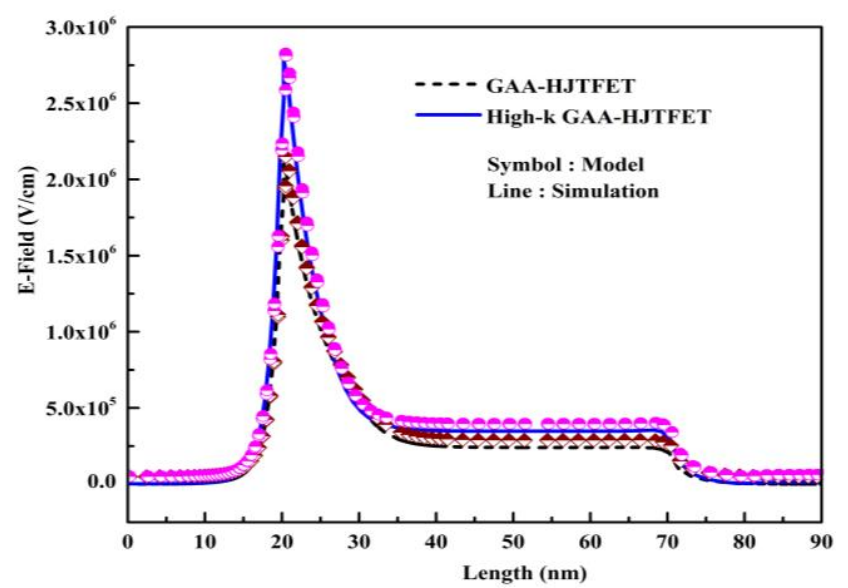

Fig. 6. Comparison plot of Electric Field profile along the channel

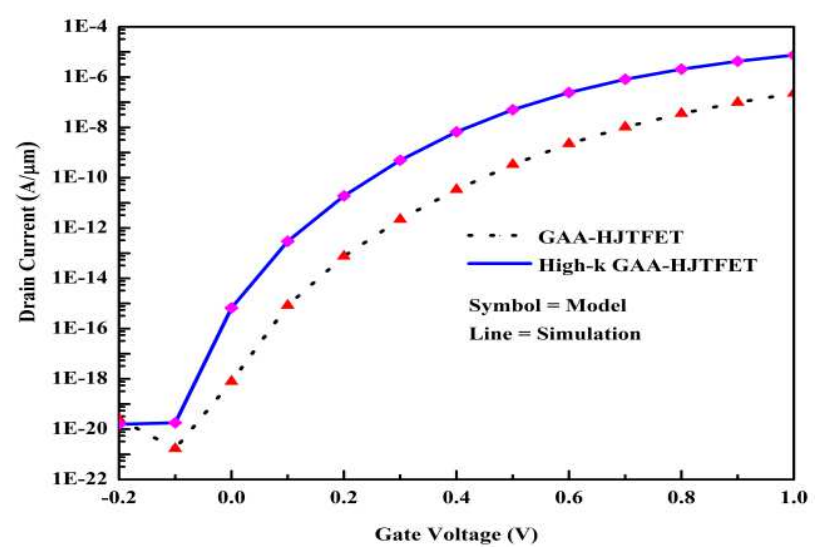

Fig. 7. Comparison $I_{D}$ vs $V_{G S}$ with and without high-k GAA-HJTFET

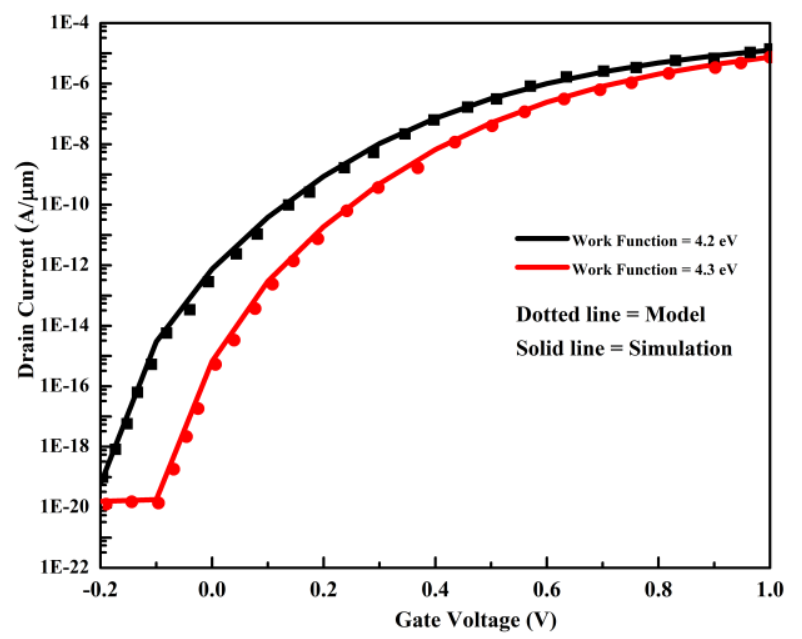

Fig. 8. $I_{D}$ vs $V_{G S}$ for different work function 


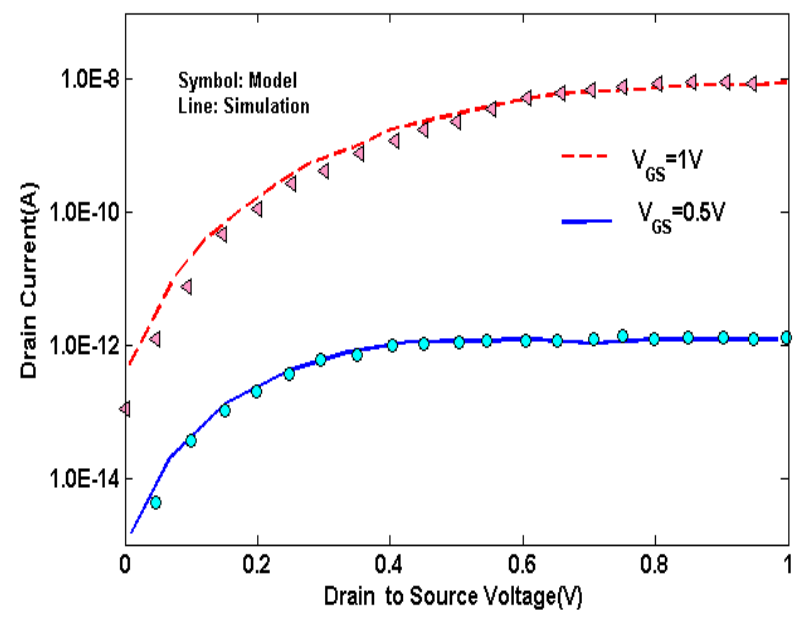

Fig. 9. $I_{D}$ vs $V_{D S}$ for the proposed device at different $\mathrm{V}_{\mathrm{GS}}$

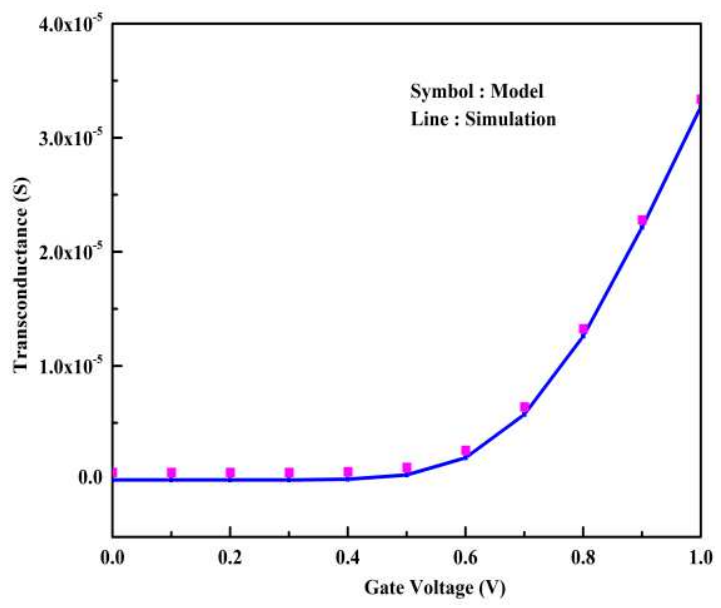

Fig. 10. $\mathrm{g}_{\mathrm{m}} \mathrm{vs} \mathrm{V}_{\mathrm{GS}}$ of the proposed device

Fig. 8. depicts the drain current variations with gate voltage for different work functions of $4.2 \mathrm{eV}$ and $4.3 \mathrm{eV}$. It is observed that OFF-state current is low and ON-state current is high, thus the $\mathrm{I}_{\mathrm{ON}} / \mathrm{I}_{\mathrm{OFF}}$ ratio is high of $10^{15}$. Fig. 9. shows the drain current variation with drain voltage for different gate voltages of $0.5 \mathrm{~V}$ and $1 \mathrm{~V}$. It represents that higher the gate to source voltage higher the drain current. Fig.10. shows the transconductance variation with the gate voltage. The transconductance is high for the high-k stacked GAA-HJTFET which in turn increases the sensitivity of the device operation.

\section{Conclusion}

In this paper, analytical modeling of high-k stacked GAA-HJTFET is developed. First the electrostatic potential modeling of high-k GAA-HJTFET is considered. To enhance the performance and reliability of model compared to previously reported devices the charge carriers across the depletion regions and channel were taken into account. The analysis is accomplished considering three depletion regions such as channel, source and drain. The analytical modeling in the channel is derived using the superposition technique, in which 1-D Poison's and 2-D Poison's equations are considered. The modeling in the source region is derived using 2-D Poison's equation with the parabolic approximation techniques. The drain region is modeled using the 1-D Poison's equation. The potential distribution modeling is used to develop the lateral electric field, minimum tunneling length and current of the model proposed. The drain current is modeled using the Kane's Model, in which the drain current is derived by integrating the BTBT generation rate. The results plotted showed the excellent match with the simulation results. The model suggested is appropriate for the low-power VLSI applications.

\section{Appendix A}

Using the continuity of lateral electric field across the source/channel interface, the potential $\varphi_{0 S}$ is expressed as

$\left.\frac{d \phi_{S}(z)}{d z}\right|_{z=0}=\left.\frac{\partial \varphi_{C}\left(z, r=r_{0}\right)}{\partial x}\right|_{z=0}$

From equation (A1), we obtain 


$$
\begin{aligned}
& -\frac{1}{k_{d}}\left(\phi_{0}+V_{T} \ln \left(\frac{N_{A}}{n_{i}}\right)\right) \sinh \left(\frac{L_{1}}{k_{d}}\right) \\
& =\frac{\lambda}{r_{0}}\left(C_{0}-C_{1}\right) J_{0}\left(\lambda \cdot r_{0}\right)
\end{aligned}
$$

Using the continuity of lateral electric field across the drain/channel interface, the potential $\varphi_{0 D}$ is expressed as

$$
\left.\frac{\partial \varphi_{C}\left(z, r=r_{0}\right)}{\partial z}\right|_{z=L_{2}}=\left.\frac{d \varphi_{D}(z)}{d z}\right|_{z=L_{2}}
$$

From equation (A3) we obtain

$$
\begin{aligned}
& \frac{V_{F, D}-\varphi_{0 D}}{L_{3}}+\frac{q N_{D} L_{3}}{2 \varepsilon_{s i}} \\
& =\frac{\lambda}{r_{0}}\left(C_{0} \exp \left(\frac{\lambda L_{2}}{r_{0}}\right)-C_{1} \exp \left(-\frac{\lambda L_{2}}{r_{0}}\right)\right) J_{0}\left(\lambda \cdot r_{0}\right)
\end{aligned}
$$

Further simplifying using (A2) and (A4) the $\varphi_{0 S}$ and $\varphi_{0 D}$ are solved to obtain expressions.

\section{Appendix B}

Substituting potential model equation (20) in the boundary condition equation (17), we get

$$
\sum_{m=1}^{\infty}\left(C_{0}+C_{1}\right) J_{0}\left(\frac{\lambda_{m}}{r_{0}} r\right)=\varphi_{0 S}-\varphi_{1 D}(r)
$$

The above equation is solved by FourierBessel series

$$
C_{0}+C_{1}=\frac{1}{N}\left(\varphi_{0 S} \frac{J_{1}(\lambda)}{\lambda}-V_{a}\right)
$$

Where

$$
V_{a}=\frac{1}{r_{0}^{2}} \int_{0}^{r_{0}} r r \varphi_{1 D}(r) . J_{0}\left(\frac{\lambda}{r_{0}} \cdot r\right) \cdot d r
$$

Similarly using boundary condition equation (18) we get

$$
\begin{aligned}
& \sum_{m=1}^{\infty}\left[C_{0} \exp \left(\frac{\lambda_{m} L_{2}}{r_{0}}\right)+C_{1} \exp \left(-\frac{\lambda_{m} L_{2}}{r_{0}}\right)\right] \times J_{0}\left(\frac{\lambda_{m}}{r_{0}} r\right) \\
& =\varphi_{0 D}-\varphi_{1} D
\end{aligned}
$$

and

$$
C_{0} \exp \left(\frac{\lambda L_{2}}{r_{0}}\right)+C_{1}\left(-\frac{\lambda L_{2}}{r_{0}}\right)=\frac{1}{N}\left(\varphi_{0 D} \frac{J_{1}(\lambda)}{\lambda}-V_{a}\right)
$$

The $C_{0}$ and $C_{1}$ can also be obtained from (B2) and (B5)

\section{References}

[1] Kumar, M., Jit, S.: A novel four-terminal ferroelectric tunnel FET for quasi-ideal switch. IEEE Trans. Nanotechnol. 14, 600602 (2015)

[2] Kumar, M., Jit, S.: Effects of electrostatically doped source/drain and ferroelectric gate oxide on subthreshold swing and impact ionization rate of strainedSi-on-insulator tunnel field-effect transistors. IEEE Trans. Nanotechnol. 14, 597-599 (2015)

[3] Nagavarapu, R., Jhaveri, R., Woo, J.C.S.: The tunnel source (PNPN) n-MOSFET: a novel high-performance transistor. IEEE Trans. Electron Devices 55, 1013-1019 (2008)

[4] Gholizadeh, M., Hosseini, S.E.: A 2-D analytical model for doublegate tunnel FETs. IEEE Trans. Electron Devices 61, 14941500 (2014)

[5] Kumar, M.J., Janardhanan, S.: Dopingless tunnel field effect transistor: design and investigation. IEEE Trans. Electron Devices 60, 3285-3290 (2013)

[6] Saurabh, S., Kumar, M.J.: Novel attributes of a dual material gate nanoscale tunnel field-effect transistor. IEEE Trans. Electron Devices 58, 404-410 (2011)

[7] Choi, W.Y., Park, B.G., Lee, J.D., Liu, T.J.K.: Tunneling field-effect transistors (TFETs) with subthreshold swing (SS) less 
than $60 \mathrm{mV} / \mathrm{dec}$. IEEE Electron Device Lett. 28, 743-745 (2007)

[8] Bhuwalka, K.K., Schulze, J., Eisele, I.: Scaling the vertical tunnel FET with tunnel bandgap modulation and gate work function engineering. IEEE Trans. Electron Devices 52, 909-917 (2005)

[9] Lee, M., Jeon, Y., Jung, J.C., Koo, S.M., Kim, S.: Feedback and tunneling operations of a p+-i-n+ silicon nanowire field-effect transistor. Nanotechnology 29, 43 (2018)

[10] Moselund, K.E., Bjork, M.T., Schmid, H., Ghoneim, H., Karg, S., Lortscher, E., Riess, W., Riel, H.: Silicon nanowire tunnel FETs: low-temperature operation and influence of high-gate dielectric. IEEE Trans. Electron Devices 58, 2911-2916 (2011)

[11] Usha, C., Vimala, P.: A tunneling FET exploiting in various structure and different models: a review. Int. Conf. Innov. Inf. Embedded Commun. Syst. 6, 72-76 (2015)

[12] Usha, C., Vimala, P.: Analytical drain current model for fully depleted surrounding gate TFET. J. Nano Res. 55, 75-81 (2018)

[13] Lu, H., Seabaugh, A.: Tunnel field-effect transistors: state-of-the-art. IEEE J. Electron Device Soc. 2, 44-49 (2014)

[14] Vishnoi, R., Kumar, M.J.: Compact analytical drain current model of gate-allaround nanowire tunneling FET. IEEE Trans. Electron Devices 61, 2599-2603 (2014)

[15] Khaveh, H.R.T., Mohammadi, S.: Potential and drain current modeling of gateall-around tunnel FETs considering the junctions depletion regions and the channel mobile charge carriers. IEEE Trans. Electron Devices 63, 5021-5029 (2016)

[16] Bagga, N., Dasgupta, S.: Surface potential and drain current analytical model of gate all around triple metal TFET. IEEE Trans. Electron Devices 64, 606-613 (2017) [17] Kumar, S., Singh, K., Goel, E., Singh, B., Kumar, M., Jit, S.: A compact 2-D analytical model for electrical characteristics of double-gate TFETs with $\mathrm{SiO} 2 /$ high-k2 stacked gate-oxide structure. IEEE Trans. Electron Devices 63, 960-968 (2016)
[18] Kumar, S., Singh, K., Chander, S., Goel, E., Singh, P.K., Baral, K.: 2-D analytical drain current model of double-gate heterojunction TFETs with $\mathrm{SiO} 2 / \mathrm{HfO} 2$ stacked gate-oxide structure. IEEE Trans. Electron Devices 65, 331-338 (2018)

[19] Zhang, L., Lin, X., He, J., Chan, M.: An analytical charge model for double gate tunnel FETs. IEEE Trans. Electron Devices 59, 3217-3223 (2012)

[20] Bardon, M.G., Neves, H.P., Puers, R., Van Hoof, C.: Pseudo-two-dimensional model for double-gate tunnel FETs considering the junctions depletion regions. IEEE Trans. Electron Devices 57, 827-834 (2010)

[21] Sze, S.: Physics of Semiconductor Devices. Wiley, New York (1981)

[22] Dash, S., Mishra, G.P.: A new analytical threshold voltage model of cylindrical gate tunnel FET(CG-TFET). Superlattices Microstruct. 86, 211-220 (2015)

[23] Ortiz-Conde, A., Garcia Sanchez, F.J., Liou, J., Cerdeira ,A., Estrada , M., Yue ,Y.: A review of recent MOSFET threshold voltage extraction methods. Microelectron. Reliab. 42, 583-596(2002)

[24] Arafat Mahmud, M., Subrina, S.: Twodimensional analytical model of threshold voltage and drain current of a double-halo gate -stacked triple material double-gate MOSFET. J. Comput.Electron.15, 525536(2016) 